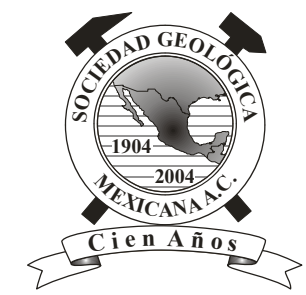

\title{
Morfogénesis, procesos y evolución del horizonte Bw cámbico en tefra- paleosuelos del volcán Nevado de Toluca
}

\author{
Carolina Jasso-Castañeda ${ }^{1, *}$, Jorge E. Gama-Castro ${ }^{2}$, Elizabeth Solleiro-Rebolledo², \\ Jaime Díaz-Ortega ${ }^{2}$ \\ ${ }^{1}$ Instituto de Investigaciones Antropológicas, Universidad Nacional Autónoma de México, Ciudad Universitaria, 04510 México, D.F. \\ ${ }^{2}$ Departamento de Edafología, Instituto de Geología, Universidad Nacional Autónoma de México, Ciudad Universitaria, 04510, D.F. \\ *karoline0105@yahoo.com.mx
}

\begin{abstract}
Resumen
La presencia de un horizonte Bw cámbico en el perfil de un paleosuelo reviste una importancia particular en las ciencias de la Tierra debido a la información ambiental y cronológica contenida en su memoria. La interpretación de esa información coadyuva a la reconstrucción paleoambiental y paleoclimática de un sitio, indicando, además, la presencia de los diferentes ciclos de estabilidad del paisaje y procesos pedológicos ocurridos durante el desarrollo del horizonte Bw. Particularmente, en este trabajo se plantea diagnosticar en una secuencia de paleosuelos: (i) el grado de desarrollo macro y micromorfológico alcanzado por los horizontes Bw; (ii) los procesos pedogenéticos básicos que propiciaron su formación; y (iii) los principales procesos tipogenéticos que determinaron su desarrollo y evolución. Para ello fueron seleccionados cuatro tefra-paleosuelos ubicados en el pedimento norte del volcán Nevado de Toluca, en las localidades denominadas como Arroyo la Ciervita (A.C.) y Zacango (Z.). Estos paleosuelos, denominados como PT1, PT2, PT3 y PT4, muestran en su perfil, horizontes Bw. Dichos horizontes se caracterizan por una composición química y mineralógica similar. Sus edades absolutas $\left({ }^{14} \mathrm{C}\right)$ oscilan de 13480 años AP a probablemente $>40000$ años AP. La metodología empleada se basa en determinar de modo cualitativo y cuantitativo los rasgos morfológicos y propiedades asociadas que son diagnósticos de los horizontes Bw. Dichas determinaciones fueron complementadas con el análisis micromorfológico, composición química elemental, así como con el uso de índices geoquímicos y relaciones químicas. Los resultados indican que los horizontes Bw presentaron un desarrollo morfopedológico que osciló de incipiente a moderado. Este desarrollo fue propiciado, inicialmente, por un intemperismo químico moderado y posteriormente por el lessivage de compuestos solubles y de partículas en suspensión, la decarbonatación, la translocación de materiales iluviales y la lixiviación moderada de bases y de hierro, manganeso y sílice. El hierro liberado formó enlaces químicos fuertes con el humus y las arcillas, generando compuestos órgano-minerales muy estables. Estos compuestos influyeron determinantemente en el desarrollo de color y estructura de los horizontes Bw. Los resultados generados permiten inferir, además, que los horizontes Bw evolucionaron en geosistemas caracterizados por presentar ciclos de estabilidad ambiental, un clima templado con variaciones en su régimen de humedad y la presencia de un bosque mixto de latifoliadas y coníferas. Bajo estas condiciones de estación y bioclimáticas, los principales procesos tipogenéticos que coadyuvaron a la evolución de los horizontes $\mathrm{Bw}$, fueron la pardificación y la andosolización.
\end{abstract}

Palabras clave: tefra-paleosuelos, Bw cámbico, índices geoquímicos, pardificación, andosolización.

\begin{abstract}
The presence of a cambic Bw horizon in a paleosol profile has a particular importance in earth sciences due to the environmental and chronological information it contains. The interpretation of that information contributes to paleoenvironmental and paleoclimatic reconstruction of the site as well as the identification of different cycles of landscape stability and pedological processes that occurred during Bw horizon development. The objectives of this paper consist in diagnosing the following aspects of a paleosol sequence: (i) the
\end{abstract}


grade of macro- and micromorphological development in the Bw horizon; (ii) the basic pedogenetic processes that favored the horizon formation; and (iii) the main typogenetic processes that determined its development and evolution. In this research, four tephra-paleosols were selected from the north pediment of the Nevado de Toluca volcano in the localities of Arroyo la Ciervita (A.C.) and Zacango (Z.). These paleosols (PT1, PT2, PT3 and PT4) have Bw horizons in their profiles. The Bw horizons have similar chemical and mineralogical compositions. Their absolute ${ }^{14} \mathrm{C}$ ages range between 13480 and probably more than 40000 years B.P. The methodology employed was based on the qualitative and quantitative determination of pedofeatures and associated properties indicative of Bw horizons. These determinations were complemented with micromorphological analyses, chemical elemental composition, and the use of geochemical indices and chemical ratios. The results indicate that the Bw horizons presented a morphopedological development ranging from incipient to moderate. At first, this development was favored by moderate chemical weathering and lessivage of soluble compounds and suspended particles, decarbonatation, translocation of illuvial materials, and moderate lixiviation of bases, iron, manganese and silica. Free iron formed strong chemical bonds with humus and clays, generating very stable organic-mineral compounds. These compounds greatly influenced color and structure development of the Bw horizons. Results also allow inferring that the Bw horizons evolved in geosystems that presented environmental stability cycles, a temperate climate with humidity oscillations, and the presence of a broadleaf-conifer mixed forest. Under these site and bioclimatic conditions, brownification and andosolization were the main typogenetic processes that participated in the Bw horizons' evolution.

Keywords: Tephra paleosols, Bw cambic, geochemical indices, brownification, andosolization.

\section{Introducción}

USDA (2006), define al horizonte cámbico, como "una capa subsuperficial de suelo, no arenosa y bien drenada, con un espesor $>15 \mathrm{~cm}$, que se caracteriza por presentar un desarrollo pedogenético que oscila de débil a moderado, así como por carecer de una acumulación apreciable de material iluvial”. En la práctica, un horizonte cámbico resulta ser cualquier sección de un perfil de suelo situado entre un horizonte A y otro subyacente relativamente inalterado (horizonte $\mathrm{C}$ ). El horizonte cámbico muestra más una estructura de suelo que de roca y un color que difiere del horizonte $\mathrm{C}$. Como se aprecia en su definición, los rasgos morfogenéticos más distintivos de un horizonte cámbico son: (i) espesor del horizonte; (ii) color; (iii) grado de desarrollo de la estructura; (iv) textura; y (v) intensidad de la iluviación. Las siglas Bw se utilizan internacionalmente para designar al horizonte cámbico.

El horizonte $\mathrm{Bw}$ es diagnóstico del grupo de suelos denominados por la WRB (2006) como Cambisoles. Sin embargo, también suele presentarse en otros edafotaxa que en ocasiones muestran una mayor evolución que los Cambisoles, por ejemplo: Andosoles y Phaeozems, así como algunos Vertisoles y Calcisoles.

Parte de la importancia de los suelos que presentan horizonte $\mathrm{Bw}$ radica, en primer término, en que regularmente muestran un alto grado de fertilidad natural, de modo particular los que se localizan en las zonas templadas (Driessen et al., 2001), y en segundo término, en que presentan a nivel mundial una amplia distribución geográfica ( $>1500$ millones de hectáreas). En México, la presencia de suelos con horizonte $\mathrm{Bw}$ resulta común en todos los relieves, y se estima que ocupan aproximadamente $30 \%$ de la superficie del territorio nacional (Cruz et al., 2007).

Desde el punto de vista científico, los suelos con horizonte Bw también revisten interés. Así, la ciencia del suelo considera a dicho horizonte como la primera etapa de transición en la evolución de un suelo joven a un suelo maduro. El horizonte B cámbico se considera el predecesor de muchos otros horizontes B más evolucionados; como el $\mathrm{B}$ argílico o el $\mathrm{B}$ espódico.

Por otra parte, las investigaciones realizadas acerca de los procesos geológicos y paleopedológicos que han propiciado la génesis y el desarrollo de los paleosuelos de México que presentan horizontes Bw también han contribuido a destacar su importancia. Entre estas investigaciones se citan las realizadas por Macías et al. (1997), Sedov et al. (2001, 2003), García-Palomo et al. (2002); Arce et al. (2003); Solleiro-Rebolledo et al. (2004); Jasso-Castañeda et al. (2002, 2006) y Jasso-Castañeda (2007). Dichas investigaciones han mostrado, directa o indirectamente, que la información contenida en la memoria del horizonte Bw es una herramienta útil que permite la reconstrucción paleoambiental de un sitio, indicando, además, la presencia de los diferentes ciclos de estabilidad del paisaje y procesos pedológicos ocurridos durante el desarrollo del horizonte. Sin embargo, resulta sorprendente que no obstante el potencial de conocimiento ambiental que puede derivarse del análisis e interpretación de la memoria del horizonte Bw, sea tan escaso el número de investigaciones y publicaciones acerca de su morfogénesis, procesos y evolución.

Con base en lo anterior, en este artículo se plantean los siguientes objetivos: (i) evaluar en una secuencia de paleosuelos el grado de desarrollo macro y micromorfológico alcanzado por los horizontes Bw; (ii) diagnosticar los procesos pedogenéticos básicos que propiciaron la formación de estos horizontes; y (iii) inferir los principales procesos tipogenéticos que determinaron su desarrollo y evolución.

Así, para este estudio, fueron seleccionados cuatro 
tefra-paleosuelos ubicados en el pedimento norte del volcán Nevado de Toluca, en las localidades denominadas como Arroyo la Ciervita (A.C.) y Zacango (Z.). Estos paleosuelos fueron denominados inicialmente como PT1 (A.C.1), PT2 (A.C.1); PT3 (A.C.3) y PT4 (Z.1). En este artículo se hace referencia a ellos como PT1, PT2, PT3 y PT4.

Los paleosuelos se formaron durante las etapas isotópicas (EI) 2 y 3 y sus edades absolutas $\left({ }^{14} \mathrm{C}\right)$ oscilan de 13480 años AP a probablemente $>40000$ años AP. La descripción detallada sobre la morfología y propiedades físicas, químicas, mineralógicas y pedoestratigráficas que caracterizan a estos paleosuelos se encuentran reportadas en Sedov et al. (2001, 2003); Solleiro-Rebolledo et al. (2004), Jasso-Castañeda et al. $(2002,2006)$ y Jasso-Castañeda (2007).

La metodología empleada para la consecución de los objetivos se basa en gran parte en criterios morfogenéticos, útiles para determinar de modo cualitativo y cuantitativo los rasgos morfológicos y propiedades asociadas que son diagnósticos de los horizontes Bw. Como se mencionó anteriormente, se utilizaron y evaluaron cinco rasgos morfológicos que de acuerdo con USDA (1984) son básicos para la diagnosis de los horizontes Bw.

Esta metodología se complementa con el análisis micromorfológico y de química elemental de los horizontes $\mathrm{Bw}$, así como con la utilización de índices geoquímicos y relaciones químicas. Todos ellos específicos para cuantificar el nivel de intemperismo, pérdida de bases y sílice, procesos tipogenéticos y grado de evolución alcanzado por dichos horizontes.

Con base en los resultados obtenidos, se ha concluido que los horizontes $\mathrm{Bw}$ se formaron bajo condiciones de estabilidad del paisaje caracterizadas por una duración moderada, así como por la acción de un paleoclima templado, aunque con variaciones en su régimen de humedad (Sedov et al., 2001, 2003; Jasso-Castañeda et al., 2002). Este paleoclima propició un intemperismo químico moderado de los horizontes $\mathrm{Bw}$, los cuales presentan porcentajes altos de minerales volcánicos fácilmente alterables, así como la presencia de una vegetación forestal mixta, la cual generó materia orgánica de fácil humificación. Ésta, junto con el hierro y las arcillas presentes, dieron origen a compuestos órgano-minerales, los cuales influyeron notablemente en el desarrollo de color y estructura de los horizontes $\mathrm{Bw}$. Todos estos factores permitieron que los principales procesos tipogenéticos involucrados en la formación de los paleosuelos y de sus horizontes Bw fueran la pardificación y la andosolización. Esta última, representada por la presencia de propiedades vítricas y ándicas, pero sin llegar a la formación de horizontes B ándico que sustituyeran a los Bw cámbico.

\section{Materiales y métodos}

\subsection{Descripción de las áreas de estudio y muestreo}

Las dos localidades en que fueron muestreados los horizontes Bw se localizan en el pedimento del volcán Nevado de Toluca, el cual se ubica geográficamente entre las coordenadas $19^{\circ} 00^{\prime} \mathrm{N}, 99^{\circ} 39^{\prime} \mathrm{O}$ y $19^{\circ} 15^{\prime} \mathrm{N}, 99^{\circ} 50^{\prime} \mathrm{O}$. (Figura 1). La localidad de Arroyo la Ciervita (A.C.) se sitúa entre los 3000 y $3015 \mathrm{~m}$ de altitud, en las coordenadas

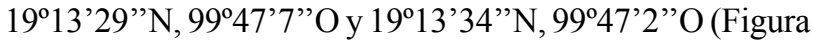
1). Los horizontes $\mathrm{Bw}$ que fueron seleccionados en este sitio para su estudio se ubican en el perfil de los tefrapaleosuelos denominados como PT1, PT2 y PT3. Dichos paleosuelos sobreyacen, respectivamente, a un flujo de ceniza retrabajada, al Flujo de Bloques y Cenizas Grises y al Flujo de Pómez Rosa definidos por Macías et al. (1997). Por otra parte, la localidad de Zacango (Z.) está situada a 2800 $m$ de altitud y se ubica entre las coordenadas $19^{\circ} 11^{\prime} 34^{\prime \prime}$

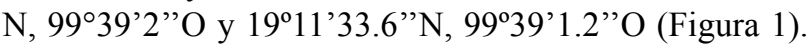
En esta localidad fue muestreado el horizonte Bw del paleosuelo PT4 que sobreyace al Flujo de Piroclastos Rosa (Macías et al., 1997).

Según la clasificación climática de Köppen modificada por García (1988), en ambas localidades se reconoce actualmente la presencia de un clima subfrío y subhúmedo (altitud de 2700-4000 m), el cual se caracteriza por una temperatura media anual que oscila entre $4{ }^{\circ} \mathrm{C}$ y $12{ }^{\circ} \mathrm{C}$ con una precipitación anual de $1100 \mathrm{~mm}$. De acuerdo con Rzedowsky (1978) y Sandoval (1987), dicho clima favorece el desarrollo de una vegetación constituida por pinos (Pinus sp., $P$. hartwegii, $P$. montezumae, $P$. pseudostrobus, $P$. ayacahuite y $P$. rudis), oyameles (Abies religiosa), ailes (Alnus sp.), cedros (Cupressus lindley) y encinos (Quercus barvinervis).

Con base en los criterios taxonómicos establecidos por WRB (2006), es posible constatar que los suelos modernos $(<10000$ años) que predominan en el área de estudio, son: Cambisoles ándicos y eútricos, Andosoles vítricos ócricos y mólicos, así como algunos Phaeozems y Regosoles téfricos. Con excepción de los Regosoles, todas las demás unidades de suelos se caracterizan por presentar un horizonte $\mathrm{Bw}$.

\subsection{Caracterización de los horizontes Bw}

La caracterización de los horizontes Bw fue hecha con base en la selección de la información generada en trabajos de investigación realizados por Jasso-Castañeda et al. $(2002,2006)$ y Jasso-Castañeda (2007). Para este trabajo, se tomaron los resultados relativos a las características morfogenéticas de los horizontes Bw, representadas por: (i) color en húmedo de los horizontes Bw (Munsell, 1975); (ii) textura (Jackson et al., 1950); (iii) espesor; 


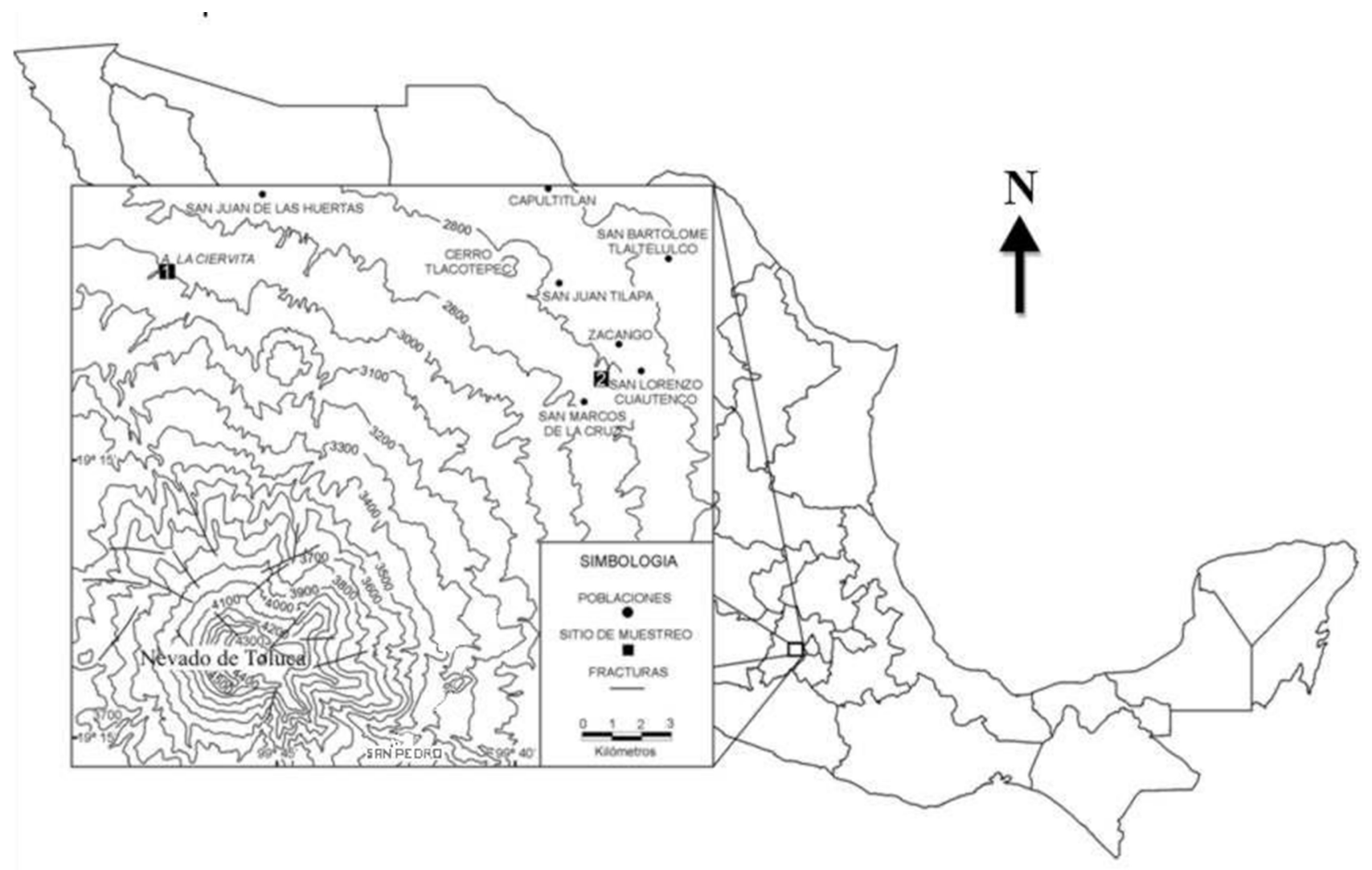

Figura 1. Localización espacial de los sitios de estudio y muestreo.

(iv) estructura; y (v) rasgos de iluviación (películas arcillosas) (Schoeneberger et al., 2002). Asimismo, fueron seleccionadas láminas delgadas de muestras inalteradas de los horizontes $\mathrm{Bw}$ estudiados. Para su descripción e interpretación se utilizó el microscopio petrográfico y se siguió el esquema y terminología propuestos por Bullock et al. (1985).

Con el objeto de llevar a cabo un análisis geoquímico de los horizontes $\mathrm{Bw}$, se consideró la composición química total determinada por medio de fluorescencia de rayos $\mathrm{X}$ (USDA, 2006). Con ella se establecieron y calcularon diferentes índices y relaciones químicas útiles para estimar el grado de intemperismo, pérdida de carbonatos, bases y sílice así como la liberación de hierro en los horizontes Bw. Dichos índices y relaciones incluyeron:

i) Índice sílice/alúmina (SA). Buen indicador del grado de intemperismo químico en rocas volcánicas y suelos (Duzgoren-Aydin et al., 2002). A medida que los valores de esta relación decrecen, la pérdida de sílice se incrementa. Se calculó a través de la siguiente fórmula: $\mathrm{SiO}_{2} / \mathrm{Al}_{2} \mathrm{O}_{3}$.

ii) Índice de alteración química (IAQ). Es un buen indicador para detectar la pérdida de $\mathrm{Ca}^{++}, \mathrm{Na}^{+}$y K $\mathrm{K}^{+}$. Se estima que a medida que los valores obtenidos de este índice aumentan, la intensidad del intemperismo se incrementa (Nesbitt y Young, 1982). Este índice se calculó por medio de la siguiente fórmula: $100 \times \mathrm{Al}_{2} \mathrm{O}_{3} /\left(\mathrm{Al}_{2} \mathrm{O}_{3}+\mathrm{CaO}+\mathrm{Na}_{2} \mathrm{O}+\mathrm{K}_{2} \mathrm{O}\right)$.

iii) Índice de Parker (IP). Índice muy sensitivo para estimar la pérdida de bases por efecto de intemperismo. Los valores más pequeños obtenidos de este índice corresponden a las muestras más desbasificadas y en ocasiones se asume que son las más viejas. Es aplicable a rocas volcánicas intermedias así como a suelos derivados de dichas rocas (Parker, 1970). Fue calculado utilizando la siguiente fórmula: $[(\mathrm{Na} / 0.35)+(\mathrm{Mg} / 0.9)+(\mathrm{K} / 0.25)+\mathrm{Ca} / 0.7)] \times 100$.

iv) Relación $\mathrm{Fe}$-ditionito/Fe-total (Fed/Fet). Generalmente, a medida que un horizonte $\mathrm{Bw}$ cámbico muestra mayor intemperismo químico, mayor desarrollo pedogenético y mayor estabilidad ambiental, el contenido de hierro cristalino (Fed) se incrementa (Malucelli et al., 1999).

v) Relación potasio/calcio. A medida que se incrementan los valores de la relación $\mathrm{K} / \mathrm{Ca}$, se refleja principalmente una mayor disolución de carbonatos. Se calculó a través de la siguiente relación elemental: $\mathrm{K}^{+} / \mathrm{Ca}^{++}$.

vi) Relación potasio/sodio. El incremento de los valores obtenidos de la relación $\mathrm{K} / \mathrm{Na}$ sugiere un aumento en la disolución de silicatos. Fue calculado a través de la siguiente relación elemental: $\mathrm{K}^{+} / \mathrm{Na}^{+}$.

\subsection{Fechamientos}

El marco cronológico de los horizontes Bw estudiados se basó en fechamientos previamente obtenidos por diferentes autores (Macías et al., 1997; Sedov et al., 2001; GarcíaPalomo et al., 2002; Solleiro-Rebolledo et al., 2004). 


\section{Resultados}

\subsection{Composición química total de los horizontes Bw}

La Tabla 1 muestra la composición química total de los horizontes Bw cámbico. Como se aprecia, todos los horizontes $\mathrm{Bw}$ exhiben una composición similar, lo cual resulta notable si se consideran las diferentes edades de los horizontes (Tabla 2). Con base en el porcentaje de $\mathrm{SiO}_{2}$ que caracteriza a los horizontes, se infiere que éstos se formaron a partir del intemperismo de un material volcánico de composición intermedia, probablemente andesítica. Los análisis petrográficos realizados por Sedov et al. (2001) denotaron una composición mineralógica que también es uniforme en todos los horizontes Bw. Éstos se caracterizaron por presentar en su fracción arena y limo, fragmentos de andesita, así como abundantes minerales primarios fácilmente intemperizables, tales como plagioclasas, hornblendas, piroxenos y vidrio volcánico (Figura 2). La presencia de estos minerales, algunos parcialmente alterados, denota la acción de un intemperismo moderado.

3.2. Características macro y micromorfogenéticas de los horizontes $\mathrm{Bw}$

En la Tabla 2 se presentan los principales rasgos morfológicos de los horizontes $\mathrm{Bw}$ cámbico y sus edades absolutas. Como ya se ha mencionado, estos rasgos son diagnósticos para la definición taxonómica de dichos horizontes e incluyen las siguientes características pedológicas:

\subsubsection{Espesor}

Todos los horizontes cumplen con el requisito de espesor diagnóstico de un B cámbico. Sin embargo, cabe mencionar que esos valores no en todos los casos son correctos, debido

Tabla 1. Composición química total de los horizontes Bw cámbico.

\begin{tabular}{ccccccccccc}
\hline $\begin{array}{c}\text { Composición } \\
\text { química } \\
\text { Paleosuelo }\end{array}$ & $\begin{array}{c}\mathrm{SiO}_{2} \\
\mathbf{\%}\end{array}$ & $\begin{array}{c}\mathrm{TiO}_{2} \\
\mathbf{\%}\end{array}$ & $\begin{array}{c}\mathrm{Al}_{2} \mathrm{O}_{3} \\
\mathbf{\%}\end{array}$ & $\begin{array}{c}\mathrm{Fe}_{2} \mathrm{O}_{3} \\
\mathbf{\%}\end{array}$ & $\begin{array}{c}\mathrm{MnO} \\
\mathbf{\%}\end{array}$ & $\begin{array}{c}\mathrm{MgO} \\
\mathbf{\%}\end{array}$ & $\begin{array}{c}\mathrm{CaO} \\
\mathbf{\%}\end{array}$ & $\begin{array}{c}\mathrm{Na}_{2} \mathrm{O} \\
\mathbf{\%}\end{array}$ & $\begin{array}{c}\mathrm{K}_{2} \mathrm{O} \\
\mathbf{\%}\end{array}$ & $\begin{array}{c}\mathrm{P}_{2} \mathrm{O}_{5} \\
\mathbf{\%}\end{array}$ \\
\hline PT1 & 59.78 & 1.155 & 22.77 & 7.288 & 0.098 & 1.791 & 3.431 & 2.813 & 1.070 & 0.112 \\
PT2 & 59.40 & 1.106 & 25.32 & 7.291 & 0.104 & 1.573 & 2.509 & 1.846 & 1.002 & 0.285 \\
PT3 & 59.05 & 0.921 & 25.46 & 6.566 & 0.087 & 1.588 & 3.203 & 2.364 & 0.894 & 0.271 \\
PT4 & 59.92 & 0.909 & 23.36 & 5.617 & 0.089 & 1.472 & 3.902 & 3.350 & 1.103 & 0.170 \\
\hline
\end{tabular}

Tabla 2. Características morfológicas diagnósticas de los horizontes Bw cámbico.

\begin{tabular}{|c|c|c|c|c|c|c|c|c|c|c|c|}
\hline \multirow[t]{2}{*}{ Unidad } & \multirow{2}{*}{$\begin{array}{l}\text { Espesor } \\
(\mathrm{cm})\end{array}$} & \multirow{2}{*}{$\begin{array}{c}\text { Color } \\
\text { (húmedo) }\end{array}$} & \multicolumn{3}{|c|}{ Estructura } & \multicolumn{3}{|c|}{ Textura } & \multicolumn{2}{|c|}{$\begin{array}{l}\text { Películas } \\
\text { arcillosas }\end{array}$} & \multirow{2}{*}{$\begin{array}{c}\text { Edad }^{14} \mathrm{C} \\
(\text { años AP) }\end{array}$} \\
\hline & & & $\mathrm{F}$ & $\mathrm{T}$ & $\mathrm{D}$ & A & $\mathrm{L}$ & $\mathrm{C}$ & $\mathrm{E}$ & $\mathrm{P}$ & \\
\hline & & $10 \mathrm{YR} 3 / 4$ & gr & $f-m-g$ & 2 & 46.0 & 15.0 & 39.0 & & & \\
\hline PT1 & 35 & $\begin{array}{c}\text { Pardo } \\
\text { amarillento } \\
\text { oscuro }\end{array}$ & sbk & $f-m$ & $2-1$ & \multicolumn{3}{|c|}{ Arcilla arenosa } & tn & $\mathrm{f}$ & $13480+/-40$ \\
\hline & & $10 \mathrm{YR} 2 / 2$ & sbk & $m-f$ & 2 & 39.0 & 28.0 & 33.0 & & & \\
\hline PT2 & 30 & $\begin{array}{l}\text { Pardo muy } \\
\text { oscuro }\end{array}$ & abk & $m-f$ & 2 & \multicolumn{3}{|c|}{ Migajón arcilloso } & tn & $\mathrm{f}$ & $27900+/-500$ \\
\hline PT3 & 40 & $\begin{array}{c}\text { 10YR3/4 } \\
\text { Pardo } \\
\text { amarillento } \\
\text { oscuro }\end{array}$ & sbk & $\mathrm{m}$ & $1-2$ & \multicolumn{3}{|c|}{$\begin{array}{c}\text { Migajón } \\
\text { arcilloso-arenoso }\end{array}$} & tn & $\mathrm{f}$ & $35650+/-1200$ \\
\hline & & $10 Y R 3 / 2$ & sbk & $f-m$ & $1-2$ & 49.0 & 26.0 & 24.0 & & & \\
\hline PT4 & 27 & $\begin{array}{l}\text { Pardo grisáceo } \\
\text { muy oscuro }\end{array}$ & sbk & $f-m$ & $1-2$ & $\begin{array}{r}\mathrm{N} \\
\text { arcillc }\end{array}$ & $\begin{array}{l}\text { ligajó } \\
\text { so-ar }\end{array}$ & noso & tn-mtk & $\mathrm{c}$ & $>40000$ \\
\hline
\end{tabular}

Estructura: $\mathbf{F}=$ forma: $\mathrm{gr}=$ granular, abk = bloques angulares, sbk = bloques subangulares; $\mathbf{T}=$ tamaño: $\mathrm{f}=$ fino, $\mathrm{m}=$ medio, $\mathrm{g}=$ grueso; $\mathbf{D}=$ desarrollo: 1 = débil, 2 = moderado. Textura: $\mathbf{A}=$ arena, $\mathbf{L}=$ limo, $\mathbf{C}=$ arcilla. Películas arcillosas: $\mathbf{E}=$ espesor: $\mathrm{tn}=$ delgado, $\mathrm{mtk}=$ moderadamente grueso; $\mathbf{P}=$ frecuencia: $\mathrm{f}=$ escasas, $\mathrm{c}=$ comunes. 


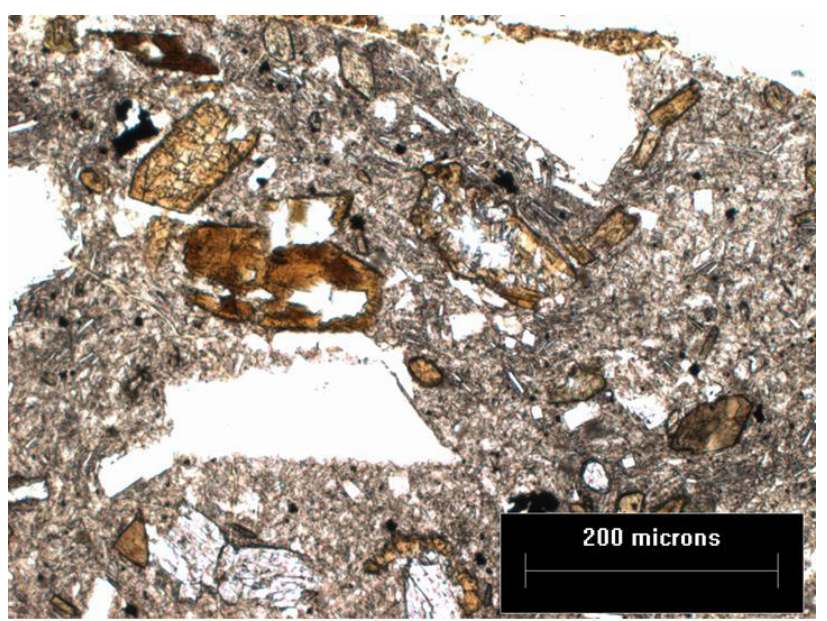

Figura 2. La composición petrográfica y mineralógica del material parental, como fue observado en láminas delgadas de horizontes $\mathrm{BC}$ y C; es bastante uniforme en todos los perfiles de suelos del área de estudio. Existen numerosos fragmentos de rocas volcánicas, principalmente andesita con textura porfídica, los cuales están compuestos principalmente de plagioclasas, hornblendas pardas, piroxenos y vidrio volcánico.

a que durante los distintos periodos de inestabilidad del paisaje (v.g. vulcanismo) que se manifestaron en el volcán Nevado de Toluca, los paleosuelos sufrieron diferentes grados de erosión (Jasso-Castañeda et al., 2006). Dicho fenómeno influyó negativamente sobre su espesor original, lo cual, además de generar datos aproximados acerca del espesor, impide evaluar la velocidad relativa de los procesos de formación del paleosuelo y su influencia en las propiedades físicas y químicas del horizonte Bw.

\subsubsection{Color}

Cabe mencionar que, aunque es una característica morfológica susceptible de variar a través del tiempo por efecto de la diagénesis, se consideró útil su determinación e interpretación. Esto debido a que los horizontes Bw seleccionados para este artículo se caracterizan por su relativa juventud (Tabla 2). Así, su edad (Pleistoceno Tardío), aunada a condiciones ambientales, moderadamente agresivas, les permitieron conservar con poca alteración su color original (Jasso-Castañeda et al., 2002, 2006). Como se observa en la Tabla 2, todos los horizontes Bw analizados presentan una tonalidad parda, la cual oscila en su intensidad y luminosidad de parda muy oscura (PT2) a parda amarillenta (PT1 y PT3) y parda grisácea (PT4). La tonalidad parda muy oscura es indicativa de que el horizonte $\mathrm{Bw}$ durante su formación recibió importantes adiciones de materia orgánica proveniente del horizonte A. Probablemente el principal proceso que propició estas adiciones fue la faunoturbación, la cual es muy activa en los suelos forestales.

Con respecto a la tonalidad parda amarillenta que se presenta en PT1 y PT3, generalmente se debe al hierro liberado directamente de la degradación de algunas fracciones de humus de "turnover rápido" (reciclado rápido) presente en el horizonte A. El hierro como hidróxido amorfo (limonita) es lixiviado y acumulado en el horizonte Bw.

Finalmente, los colores pardo- grisáceos que caracterizan al horizonte Bw en PT4 sugieren una remoción del hierro por agentes quelatantes o por el lavado estacional con agua ácida. Ambos procesos son comunes en suelos forestales (Leirós et al., 2000).

\subsubsection{Estructura}

Las estructuras en forma de bloques subangulares y angulares son las más frecuentes en los horizontes $\mathrm{Bw}$ (Tabla 2, Figura 3). Sin embargo, también fueron observadas algunas estructuras granulares y prismáticas. En general, los agregados estructurales se caracterizan por presentar tamaños finos (1-2 mm) a medios $(>2-5 \mathrm{~mm})$. Dichos agregados se caracterizan además por mostrar diferentes grados de desarrollo, los cuales oscilan de débil a moderado. Los reportes de campo realizados por Jasso-Castañeda (2007) señalan que la estructura en bloques se presenta de modo obvio y frecuente en los horizontes Bw. En algunos casos, se observaron estructuras en bloques subangulares y granulares (PT1) y, en menor porcentaje, la presencia en el horizonte $\mathrm{BC}$ de algunos agregados prismáticos débilmente desarrollados. De acuerdo con Waltman y Ciolkosz (1995), esta intergradación de formas estructurales puede asociarse con el tipo de vegetación predominante, los patrones de distribución de raíces, contenido de materia orgánica y humedad que caracterizan el subsuelo de bosque $v s$. pradera. En los estudios clásicos realizados por Harper (1937) sobre la estructura prismática en suelos de zonas templadas, éste concluye que en las áreas de pradera se desarrollan suelos con horizontes $\mathrm{Bw}$ donde la estructura prismática es dominante sobre la estructura en bloques. En contraste, la estructura en bloques resulta frecuente en el horizonte $\mathrm{Bw}$ de perfiles forestales.

Por otra parte, Shoji et al. (1993) consideran que la alternancia de periodos de humedad-sequía ambiental y pedológica tiene un efecto notable sobre la formación de agregados estructurales. Estos autores reportan que en algunos suelos (Cambisoles-Andosoles) donde predomina en el año un déficit de humedad en el suelo (régimen ústico) se desarrolla en el horizonte A como en el Bw una estructura predominantemente granular, asociada a bloques (Figura 3). En contraste, en los suelos que presentan humedad la mayor parte del año (régimen údico) se muestra una estructura en bloques, principalmente subangulares y angulares.

\subsubsection{Textura}

La Tabla 2 muestra que los horizontes Bw se caracterizan por presentar clases texturales finas, que oscilan de arcilla arenosa a migajón arcillo-arenoso. La distribución del tamaño de partícula en estos horizontes se caracteriza por las siguientes variaciones: la arena oscila de $39 \%$ a $46 \%$, el limo de $15 \%$ a $28 \%$ y la arcilla de $22 \%$ a $39 \%$ (Tabla 2).

Es probable que esas fluctuaciones se deban principalmente a diferencias cronológicas en el depósito y 


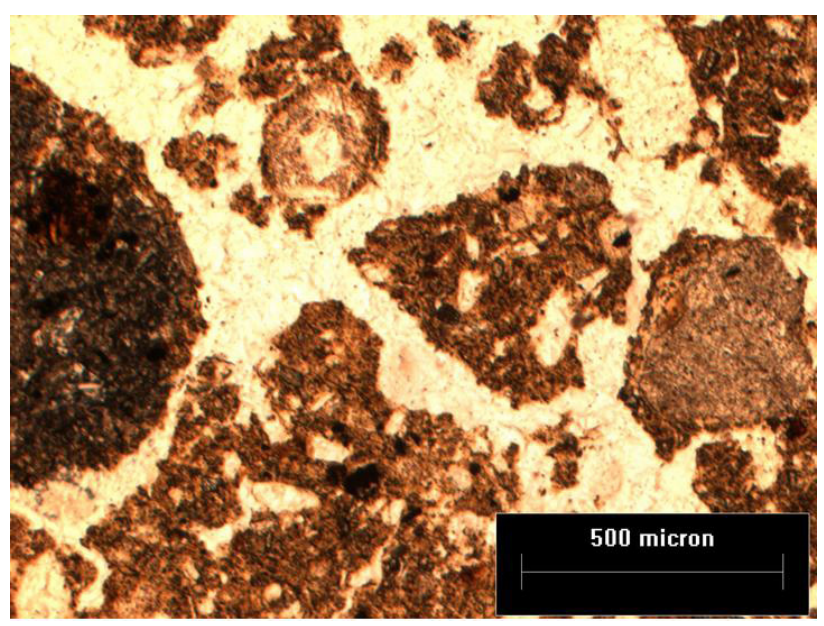

Figura 3. Estructura compleja. En el horizonte Bw del paleosuelo PT1, la estructura es compleja: existen gránulos de diferente tamaño asociados con bloques subangulares. El material fino es isotrópico, pardo claro y consiste en arcilla asociada con óxidos de hierro.

asimismo a la composición textural del material volcánico que originó los suelos, la cual, en parte, fue heredada a los horizontes Bw.

Así, en el caso de PT1 y PT2, que se formaron a partir de los ciclos de actividad volcánica mas reciente, el horizonte $\mathrm{Bw}$ exhibe el mayor contenido de arcilla (Tabla 2). En contraste, los horizontes Bw de PT3 y PT4, que se presentan en los suelos más antiguos, son los que menor contenido de arcilla muestran (Tabla 2).

\subsubsection{Rasgos de iluviación: películas arcillosas}

Las observaciones macro y micromorfológicas de los agregados estructurales presentes en los horizontes $\mathrm{Bw}$ permitieron detectar en PT1, PT2 y PT3 la existencia, escasa, de rasgos de iluviación, así como de algunos pocos rasgos relacionados con procesos reductomórficos, entre ellos, nódulos ferruginosos y moteado, los cuales fueron ampliamente descritos por Sedov et al. (2001).

Los rasgos de iluviación están representados por revestimientos de hierro (Figura 4) y por películas de arcilla, las cuales en el caso de PT4 son comunes, aunque no suficientes para constituir un horizonte B argílico (Figura 5).

Con excepción de PT4, donde algunas películas de arcilla son moderadamente gruesas $(0.07-0.09 \mathrm{~mm})$, en los demás horizontes $\mathrm{Bw}$ éstas se caracterizan por un espesor delgado $(<0.05 \mathrm{~mm})$, baja abundancia $(<1 \%)$ y su localización casi exclusivamente en poros.

Con base en el espesor, frecuencia y ubicación de las películas arcillosas, se estima que estos rasgos denotan la presencia de procesos de iluviación de arcilla muy débiles en PT1, PT2 y PT3 y débiles a moderados en PT4. Empero, en todos los casos, dichos rasgos confirman la presencia de iluviación en los horizontes $\mathrm{Bw}$ estudiados.

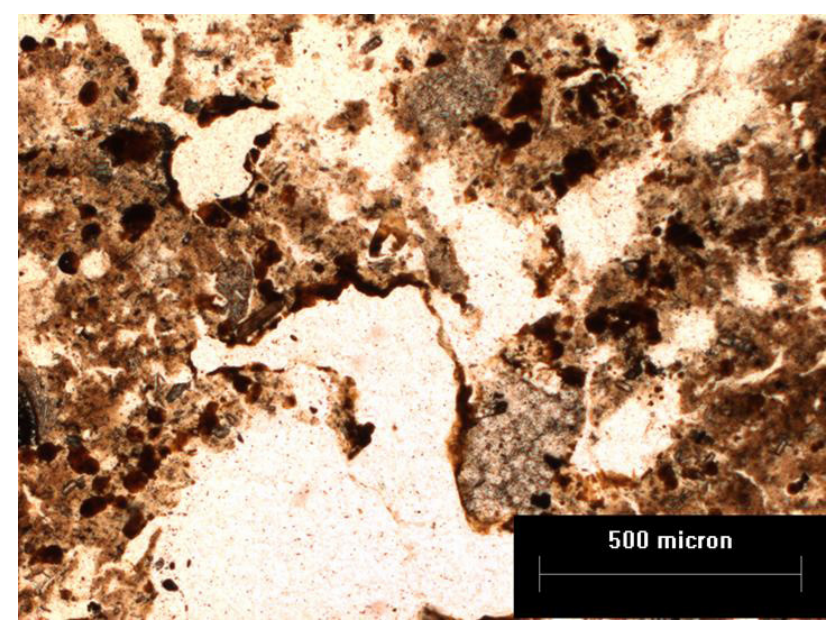

Figura 4. Los rasgos de iluviación en PT3 están representados por revestimientos de hierro y por escasas y delgadas películas de arcilla.

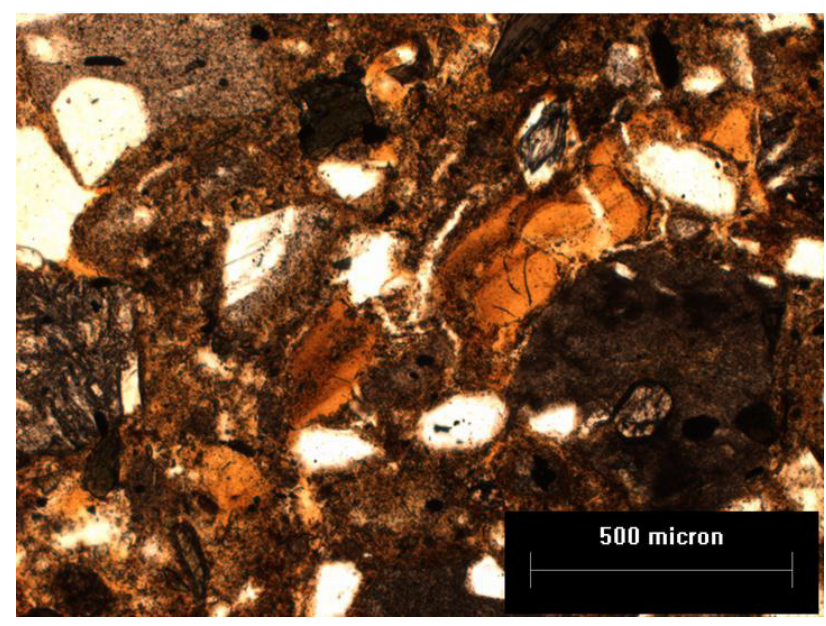

Figura 5. Aún cuando la morfología del perfil del paleosuelo PT4 es similar a la de PT2 y PT3, éste se distingue por presentar algunos rasgos micromorfológicos: cutanes de arcilla, los cuales fueron observados tanto en el horizonte Ah como también en el Bw. Sin embargo, la cantidad total de estos cutanes no es suficiente para constituir un horizonte Bt argílico.

3.3. Índices geoquímicos y relaciones químicas características de los horizontes $\mathrm{Bw}$

\subsection{1. Índice sílice/alúmina ( $S A)$}

Se estima que a medida que los valores de esta relación decrecen, la pérdida de sílice se incrementa. Sin embargo, este índice también decrece cuando un intemperismo ligero propicia la lixiviación de la sílice durante un ciclo largo de estabilidad ambiental.

La Tabla 3 muestra, respecto a los valores del índice SA, una secuencia de intemperismo que denota un orden en su intensidad. Esta secuencia puede ser representada de la siguiente manera: PT2 > PT3 > PT1 > PT4. Así, PT2 es el horizonte más intemperizado y PT4 el menos alterado. 
Tabla 3. Índices geoquímicos y relaciones químicas de los horizontes Bw cámbico.

\begin{tabular}{lccccccc}
\hline $\begin{array}{l}\text { Indices y } \\
\text { relaciones }\end{array}$ & $\mathrm{SA}$ & $\mathrm{IAQ}$ & $\mathrm{IP}$ & $\mathrm{SiO}_{2} / \mathrm{R}_{2} \mathrm{O}_{3}$ & $\mathrm{Fed} / \mathrm{Fet}$ & $\mathrm{K} / \mathrm{Ca}$ & $\mathrm{K} / \mathrm{Na}$ \\
\hline PT1 & 4.46 & 65.41 & 0.31 & 3.6 & 0.24 & 0.31 & 0.38 \\
PT2 & 3.9 & 74.44 & 0.24 & 3.28 & 0.33 & 0.4 & 0.54 \\
PT3 & 3.94 & 70.42 & 0.27 & 3.31 & 0.26 & 0.28 & 0.4 \\
PT4 & 4.56 & 62.84 & 0.34 & 3.7 & 0.15 & 0.28 & 0.33 \\
\hline
\end{tabular}

SA: relación sílice/alúmina; IAQ: índice de alteración química; IP: Índice de Parker; Fed/Fet: hierro extraído con ditionito-citrato-bicarbonato/ hierro total.

Dicha secuencia probablemente resultó tanto de la duración de los ciclos de estabilidad como del paleoclima en que se formaron los paleosuelos. Por ejemplo, PT1, que muestra un valor alto de SA, se formó hace 13480 años AP, durante la época más fría y seca del Pleistoceno Tardío a nivel global (Caballero et al., 2010). Dichas condiciones limitaron el grado de intemperismo, así como su desarrollo pedológico (Solleiro-Rebolledo et al., 2004).

En el caso de PT2, que presenta el valor más bajo de SA (Tabla 3), éste se formó durante la EI-2, antes del Último Máximo Glacial. Jasso-Castañeda (2007) propone que durante la formación de PT2 existió un clima templado más húmedo que el actual, lo que propició un mayor intemperismo. Los análisis elaborados por Sedov et al. (2003) y Jasso-Castañeda (2007) muestran la presencia de cantidades significativas de humus y alofano en los horizontes A y Bw de PT2. Su presencia, según Sedov et al. (2003), denota la existencia de condiciones significativas de humedad.

En contraste, PT4 se caracteriza por presentar el valor más alto de SA. Se considera que esta unidad se formó en un clima templado, pero más seco que el actual (Sedov et al., 2003). En este caso, la existencia de películas arcillosas en el horizonte Bw de PT4 (Tabla 2), sugiere la presencia de un periodo de estabilidad prolongado para el desarrollo de estos rasgos.

\subsection{2. Índice de Alteración Química (IAQ)}

Duzgoren-Aydin et al. (2002) establecieron que los valores de IAQ, cuando oscilan entre 50 y 60, indican la presencia de un intemperismo incipiente; los valores que fluctúan entre 60 y 80 son característicos de un intemperismo intermedio; y cuando son mayores a 80, denotan la presencia de un intemperismo extremo. En contraste, los valores menores a 50 son típicos de una roca muy alterada, pero que aún no constituye un horizonte de suelo.

Como se aprecia en la Tabla 3, todos los valores de IAQ muestran un grado de intemperismo intermedio. Sin embargo, el valor de PT4 apenas rebasa los límites establecidos para un intemperismo incipiente, siendo PT2 y PT3 los que presentan los valores más altos.

Los valores obtenidos para IAQ son totalmente congruentes con los valores reportados de SA y, asimismo, con la secuencia de intemperismo propuesta para dichos valores.

\subsection{3. Índice de Parker (IP)}

Presenta una función similar al índice IAQ, salvo que para su cálculo sólo se utilizan proporciones atómicas de elementos móviles, incluyendo $\mathrm{Mg}^{++}$. Estas características permiten que el índice sea recomendable para medir la movilidad, acumulación y pérdida de bases a través de los horizontes de perfil (Souri et al., 2006).

En este caso, se utilizó el índice IP para medir el grado de pérdida de bases alcanzado por los horizontes Bw, mostrando una tendencia a disminuir sus valores conforme se incrementaron las pérdidas de $\mathrm{Ca}^{++}, \mathrm{Mg}^{++} \mathrm{Na}^{+}$y K $\mathrm{K}^{+}$debido al intemperismo. Como se observa en la Tabla 3, los valores más bajos de IP corresponden con los horizontes Bw más lixiviados e intemperizados (PT2 y PT3), en tanto que los valores más altos se presentan en los horizontes Bw menos intemperizados (PT1 y PT4).

\subsubsection{Relación Fe-ditionito/Fe-total (Fed/Fet)}

Este índice refleja el grado en que el hierro contenido en los minerales primarios del suelo se transforma a óxidos de Fe (III) con un alto nivel de cristalinidad. En la formación de dichos óxidos, se incluyen principalmente la intensidad del intemperismo y la duración de la estabilidad ambiental. Sin embargo, algunos autores consideran que al aumentar la edad de un suelo, también se incrementa la cantidad de óxidos de hierro cristalino (Schwertmann y Latham, 1986; Schwertmann, 1988).

Como se aprecia en la Tabla 3 , los valores respecto al índice Fed/Fet oscilan de moderados $(0.33)$ a bajos (0.15). También es posible notar que los valores de este índice generan una secuencia que muestra que la mayor acumulación de óxidos de Fe (III) cristalino se detectó en PT2 y la menor en PT4. Esta secuencia también es totalmente congruente con la secuencia de intemperismo que se presenta para SA. En este caso no existe ninguna relación entre la concentración de los óxidos y la edad absoluta de los horizontes Bw (Tabla 2).

\subsubsection{Relación potasio/calcio}

Este índice estima la movilización del $\mathrm{Ca}^{++}$y de los carbonatos. En general, los horizontes Bw que se presentan en este artículo se caracterizan por sus contenidos moderados a bajos de calcio (Tabla 2). Sin embargo, la decarbonatación propiciada por una débil disolución y lessivage fue un proceso pedogenético fundamental en la etapa inicial de la formación de los horizontes Bw. Así la decarbonatación favoreció que pequeños porcentajes de arcillas pudieran ser dispersadas, transportadas por el agua y acumuladas en el horizonte Bw. Los valores más altos de esta relación fueron identificados en el horizonte $\mathrm{Bw}$ de PT2, resultando valores parecidos en los demás horizontes.

\subsubsection{Relación potasio/sodio}

La relación $\mathrm{K} / \mathrm{Na}$ fue usada para indicar el grado de 
intemperismo de los silicatos como son los feldespatos. Se interpreta que al incrementarse los valores de la relación $\mathrm{K}$ / $\mathrm{Na}$, aumenta la disolución de silicatos. La Tabla 3 muestra la absoluta correlación que existe entre la disolución de los silicatos y la intensidad de intemperismo que presentan los horizontes Bw. Así, el horizonte Bw de PT2 es el más intemperizado y también es el que presenta el valor de $\mathrm{K} / \mathrm{Na}$ más alto. En contraste, PT4 representa al horizonte menos intemperizado y con el valor de disolución de silicatos más bajo.

\section{Discusión}

Con base en los resultados obtenidos en este estudio, se infiere que la formación, desarrollo y evolución de los horizontes $\mathrm{Bw}$ es resultado de un ciclo de intemperismo moderado, asociado a procesos básicos y tipogenéticos como la pardificación y la andosolización.

El proceso de pardificación consiste de los siguientes procesos básicos: (i) lessivage de los carbonatos y lixiviación de bases alcalinas y alcalinotérreas; (ii) iluviación incipiente de arcilla; (iii) liberación del hierro como consecuencia del intemperismo de los minerales primarios; y (iv) formación de (oxi)-hidróxidos férricos y ferrosos hidratados, parcialmente cristalinos y predominantemente amorfos. Estos procesos facilitan la unión del hierro libre con las arcillas y el humus, formando complejos órgano-minerales, los que en su mayoría se caracterizan por su color pardo.

Para que estos procesos se presenten y la pardificación ocurra, resulta necesario la conjunción de las siguientes variables: (a) presencia de un material parental rico en minerales fácilmente intemperizables; (b) contenidos altos en el suelo de materia orgánica de fácil humificación (mull); (c) ciclos de estabilidad del paisaje con una duración moderada; y (d) un clima templado.

De acuerdo con los datos obtenidos, los tefra-paleosuelos estudiados reúnen estas variables, estimándose que en la zona de estudio prevaleció un paleoclima predominantemente templado, aunque con variaciones en su régimen de humedad ambiental y pedológico. Estas variaciones propiciaron la formación de intergrados entre Cambisoles (PT1) y Andosoles (PT2, PT3, PT4)

El proceso de pardificación resulta particularmente evidente en PT1, el cual se formó en un periodo caracterizado por condiciones climáticas menos húmedas que las que prevalecieron durante la génesis del resto de las unidades. PT1 se desarrolló durante la EI-2, en el Glacial Terminal, que en el centro de México se caracteriza por el retorno a condiciones más cálidas con un retroceso marcado en los glaciares (Caballero et al., 2010). Dicho clima, favoreció la formación de haloisita por la deshidratación del alofano.

PT3 se formó durante la EI-3. Sedov et al. (2003) y Yakimenko et al. (2007) señalan que los análisis realizados a estos tefra-paleosuelos denotaron la presencia de un contenido significativo de humus y alofano que caracteriza a los horizontes A y Bw del paleosuelo. Esto, junto con la presencia de plantas $\mathrm{C} 3$, refleja paleocondiciones de humedad similares a las actuales. Dichas condiciones favorecieron la hidrólisis de los materiales volcánicos (tefra) y la formación de óxidos amorfos, alofano y minerales microcristalinos de hierro, aluminio y sílice distribuidos en el perfil del suelo con bastante regularidad. Estas características son diagnósticas del proceso de andosolización (Shoji et al., 1993). PT4, formado también durante EI-3, refleja condiciones de mayor sequía, dado el menor grado de alteración que presenta. Esto es congruente con las observaciones de Sedov y colaboradores (2003) basadas en otros indicadores.

El paleosuelo PT2 se formó a finales de la EI-3 y durante la EI-2. A diferencia de PT3 y PT4, éste presenta un grado de intemperismo más fuerte, lo que se interpreta como condiciones intempéricas más agresivas que las actuales.

Los rasgos pedológicos derivados de la andosolización tendieron a sobreponerse a los rasgos derivados del proceso de pardificación. Sin embargo, debido probablemente a que las condiciones de estabilidad ambiental fueron insuficientes en su duración, ninguno de los tefra-paleosuelos (PT2, PT3 y PT4) llegó a constituir un horizonte B ándico (JassoCastañeda, 2007). Dicha limitación en tiempo sólo permitió en estos paleosuelos la presencia de propiedades vítricas y algunas ándicas, entre ellas, la presencia de minerales de rango corto (v.g. alofano). Esos minerales y complejos son comúnmente parte de una secuencia de intemperismo en depósitos piroclásticos, la cual puede representarse de la siguiente forma: material téfrico - propiedades vítricas propiedades ándicas - formación de un horizonte B ándico.

\section{Conclusiones}

\subsection{Composición y características macro y micromorfológicas de los horizontes $\mathrm{Bw}$}

a. No obstante que todos los horizontes Bw muestran diferencias significativas en su edad absoluta, en su conjunto presentan una composición química y mineralógica similar. El análisis micromorfológico denota que la fracción limo y arena en los horizontes Bw está constituida por líticos y minerales fácilmente intemperizables, entre ellos plagioclasas, hornblendas, piroxenos y vidrio volcánico.

b. En todos los horizontes Bw se cumple con el valor de espesor requerido taxonómicamente para ser clasificados como Bw cámbico. Sin embargo, en la práctica dichos valores resultan inciertos, debido a los procesos de erosión que se presentaron periódicamente, los cuales afectaron la profundidad de los paleosuelos y el grosor de sus horizontes.

c. La tonalidad parda (croma), que predomina en los horizontes $\mathrm{Bw}$, es una propiedad cromática derivada de la unión del hierro libre con las arcillas y el humus, formando complejos órgano-minerales.

d. Con excepción de PT1, las estructuras en formas 
de bloques angulares, subangulares y, en muy escasa proporción, prismáticas son las más comunes en los horizontes Bw. En el caso de PT1, se detectó en un estudio micromorfológico que su estructura es más compleja, ya que está constituida por agregados granulares de diferentes tamaños, asociada con bloques subangulares.

e. Las láminas delgadas de los horizontes Bw de PT2 y PT3 muestran al microscopio que en la base de dichos horizontes existen evidencias de compactación, así como algunos rasgos relacionados con procesos reductomórficos. Estos rasgos están representados por nódulos ferruginosos y moteados de coloración ocre.

f. En general, los horizontes Bw presentan clases texturales finas, lo cual cumple con los requisitos taxonómicos de un horizonte cámbico. Las fluctuaciones en el tamaño de partículas que muestran entre ellos se deben a diferencias cronológicas en el depósito y composición textural del material volcánico que originó a los paleosuelos, así como también a la probable evolución poligenética de los materiales que fue característica de la zona.

g. El análisis micromorfológico de los horizontes Bw mostró que con excepción de PT4 los rasgos de iluviación en los horizontes Bw están incipientemente representados por escasos cutanes y películas de arcilla. En PT4, se observa la presencia de frecuentes películas de arcilla de espesor delgado a moderado que se localizan principalmente cerca de los poros. Sin embargo, el porcentaje total de estas películas no llega a constituir un horizonte Bt argílico.

\section{2. Índices geoquímicos y relaciones químicas} características de los horizontes $\mathrm{Bw}$

a. Los índices geoquímicos, así como las relaciones químicas utilizadas en este estudio para evaluar al intemperismo y los procesos básicos de alteración asociados a él, mostraron resultados congruentes entre sí. Éstos coinciden al indicar la presencia de un intemperismo moderado que interactuó en todos los horizontes Bw. Sin embargo, los valores que reportan los índices SA y IAQ muestran que, aunque el nivel de intemperismo fue moderado, no se presentó con la misma intensidad en todos los horizontes Bw. Esta variación en la intensidad dio origen a una secuencia de meteorización, la cual puede ser representada de la siguiente manera: PT2 $>$ PT3 $>$ PT1 $>$ PT4.

b. Considerando que la distribución de los paleosuelos en el perfil de la pendiente, así como composición química y mineralógica de los horizontes Bw, son muy parecidas, se infiere que las diferencias en la magnitud de la intensidad del intemperismo que se presentan entre los horizontes $\mathrm{Bw}$ fueran propiciadas, principalmente, por la duración de los ciclos de estabilidad ambiental, así como por los distintos regímenes de humedad ambiental y pedológica que prevalecieron en cada caso.

c. Los índices y relaciones utilizados también permiten concluir lo siguiente: el intemperismo propició, a través de procesos básicos de alteración, como la disolución y el lessivage, la pérdida de $\mathrm{Ca}^{++} \mathrm{y}$ de carbonatos (relación $\mathrm{K}$ / $\mathrm{Ca}$ ). Dicha pérdida favoreció la iluviación y acumulación incipiente de películas arcillosas en los horizontes Bw. Asimismo, generó la lixiviación de bases alcalinas y alcalinotérreas (índice IP) y de sílice (índice SA).

Por otra parte, el intemperismo también facilitó la disolución de los feldespatos (relación $\mathrm{K} / \mathrm{Na}$ ) y la hidrólisis de los minerales primarios, permitiendo la liberación de varios elementos, entre ellos el hierro (Fed/Fet).

\subsection{Procesos básicos y tipogenéticos de los horizontes} $\mathrm{Bw}$

a. La formación, desarrollo y evolución de los horizontes Bw fueron resultado de: (i) ciclos de estabilidad del paisaje; (ii) un intemperismo moderado, asociado a procesos básicos de alteración; y (iii) la acción de procesos tipogenéticos como la pardificación y la andosolización.

b. En conjunto, los resultados generados también permiten inferir que los horizontes evolucionaron en geosistemas caracterizados por presentar ciclos de estabilidad ambiental, un clima templado, con variaciones en su régimen de humedad y la presencia de un bosque mixto de latifoliadas y coníferas.

\section{Agradecimientos}

Los autores agradecen el apoyo de los proyectos que financiaron la investigación: CONACYT 32337-T y G35442-T, PAPIIT, UNAM IN104600. Se agradece a Patricia Girón y Rufino Lozano del Laboratorio de Fluorescencia de Rayos X, por realizar los análisis de composición química total. También a María Antonieta Roca Rodríguez por su apoyo en la traducción al inglés del resumen.

\section{Referencias}

Arce, J.L., Macías J.L., Vázquez-Selem, L., 2003, The 10.5 Ka Plinian eruption of Nevado de Toluca volcano, Mexico: Stratigraphy and hazard implications: Geological Society of America Bulletin, 115, 230-248.

Bullock, P., Fedoroff, N., Jongerius, A., Stoops, G., Tursina, T., Babel, U., 1985, Handbook for Soil Thin Section Description: Wolverhampton, Reino Unido, Waine Research Publications, 152 p.

Caballero, M., Lozano-García, S., Vázquez-Selem, L., Ortega, B., 2010, Evidencias de cambio climático y ambiental en registros glaciales y en cuencas lacustres del centro de México durante el último máximo glacial: Boletín de la Sociedad Geológica Mexicana, 62, 359-377.

Cruz, C., Balbontin, C., Paz, F., Etchevers J., Krasilnikov, P., 2007, Los suelos de México y su geografía (en línea), en Ibáñez, J.J.: Un Universo invisible bajo nuestros pies - Los suelos y la vida: Valencia, España, Universidad de Valencia, publicado 22 de junio de 2007, disponible en <www.madrimasd.org/blogs/ universo/2007/06/22/68351> consultado 13 de febrero de 2012. 
Driessen, P., Deckers, J., Spaargaren, O., 2001, Lecture Notes on the Major Soils of the World: Roma, Food and Agriculture Organization of the United Nations, $334 \mathrm{p}$.

Duzgoren-Aydin, N.S., Aydin, A., Malpas, J., 2002, Re-assessment of chemical weathering indices: case study on pyroclastic rocks of Hong Kong: Engineering Geology, 63, 99-119.

García, E., 1988, Modificaciones al sistema de clasificación climática de Koeppen (para adaptarlo a las condiciones de la República Mexicana): Ciudad de México, Instituto de Geografía, Universidad Nacional Autónoma de México, 219 p.

García-Palomo, A., Macías, J.L., Arce, J.L., Capra, L., Garduño, V.H., Espíndola, J.M., 2002, Geology of Nevado de Toluca volcano and surrounding areas, central Mexico (Map and Chart Series MCH089): Boulder, Colorado, Geological Society of America, 1-48.

Harper, H.J., 1937, Factors which affect the development of prismatic structure in soils of the southern Great Plains: Soil Science Society of America Proceedings, 2, 447-453.

Jackson, M.L., Whittig, L.D., Pennington, A.S., 1950, Segregation procedure for the mineralogical analysis of soil: Soil Science Society of America Proceedings, 14, 77-81.

Jasso-Castañeda, C., 2007, La memoria de los paleosuelos del Nevado de Toluca: un registro de estabilidad geomórfica y cambio ambiental durante el Cuaternario tardío: Ciudad de México, Instituto de Geología, Universidad Nacional Autónoma de México, tesis de doctorado, $133 \mathrm{p}$.

Jasso-Castañeda, C., Sedov, S.N., Solleiro-Rebolledo, E., GamaCastro, J.E., 2002, El desarrollo de los paleosuelos como índice de la estabilidad del paisaje. Un ejemplo del centro de México: Investigaciones Geográficas, 47, 20-35.

Jasso-Castañeda, C., Sedov, S.N., Gama-Castro, J., Solleiro-Rebolledo, E., 2006, Los paleosuelos: índices del paleoambiente y de la estabilidad del paisaje del Nevado de Toluca: Terra Latinoamericana, 24, 151-161.

Leirós, M.C., Trasar-Cepeda, C., Seoanes, S., Gil-Sotres, F., 2000, Biochemical properties of acid soils under climax vegetation (Atlantic oakwood) in an area of the European temperate-humid zone (Galicia, NW Spain): general parameters: Soil Biology and Biochemistry, 32, 733-745.

Macías, J.L, García-Palomo, A., Arce, J.L., Siebe, C., Espíndola, J.M., Komorowski, J.C., Scott, K., 1997, Late Pleistocene-Holocene cataclysmic eruptions at Nevado de Toluca and Jocotitlán volcanoes, Central Mexico: Bringham Young University, Geology Studies, 42, 493-528.

Malucelli, F., Terribile, F., Colombo, C., 1999, Mineralogy, micromorphology and chemical analysis of andosols on the Island of Sao Miguel (Azores): Geoderma, 88, 73-98.

Munsell, 1975, Soil Color Chart: Baltimore, Maryland, Macbeth Division of Kollmorgen Corporation.

Nesbitt, H.W., Young, G.M., 1982, Early Proterozoic climates and plate motions inferred from major elementary chemistry of lutites: Nature, 299, 715-717.

Parker, A., 1970, An index of weathering for silicate rocks: Geological Magazine, 107, 501-504.

Rzedowsky, J., 1978, Vegetación de México: Ciudad de México, Limusa, $432 \mathrm{p}$.
Sandoval, B.A., 1987, Actualización y análisis cartográfico sobre usos de suelo y vegetación del Parque Nacional Nevado de Toluca, Estado de México: Facultad de Filosofía y Letras, Universidad Nacional Autónoma de México, tesis de licenciatura, 125 p.

Schoeneberger, P.J., Wysocki, D.A., Benham, E.C., Broderson, W.D, 2002, Field Book for Describing and Sampling Soils, Version 2.0: Lincoln, Nebraska, Natural Resources Conservation Service, $227 \mathrm{p}$.

Schwertmann, U., Latham M., 1986, Properties of iron oxides in some New Caledonian Oxisoles: Geoderma, 39, 105-123.

Schwertmann, U., 1988, Ocurrence and formation of iron oxides in various pedoenvironments, en Stucki, J.W., Goodman, B.A., Schwertmann, U. (eds.), Iron in Soils and Clay Minerals: Dordrecht, Países Bajos, D. Reidel Publishing, 267-308.

Sedov, S.N., Solleiro-Rebolledo, E., Gama-Castro, J.E., Vallejo-Gómez, E., González-Velázquez, A., 2001, Buried paleosols of the Nevado de Toluca: an alternative record of Late Quaternary environmental change in central Mexico: Journal of Quaternary Science, 16, 375-389.

Sedov, S.N., Solleiro-Rebolledo, E., Morales-Puente, P., Arias-Herrería, A, Vallejo-Gómez, E., Jasso-Castañeda, C., 2003, Mineral and organic components of the buried paleosols of the Nevado de Toluca/central Mexico as indicators of paleoenvironments and soil evolution: Quaternary International, 106/107, 169-184.

Shoji, S., Nanzyo, M., Dahlgren, R., 1993, Volcanic Ash Soils. Genesis, Properties and Utilization: Amsterdam, Elsevier Science Publishers, $288 \mathrm{p}$.

Solleiro-Rebolledo, E., Macías., J.L., Gama-Castro, J.E., Sedov, S.N., Sulerzhitsky, L.D., 2004, Quaternary pedostratigraphy of the Nevado de Toluca volcano: Revista Mexicana de Ciencias Geológicas, 21, 101-109.

Souri, B., Watanabe, M., Sakagami, K., 2006, Contribution of Parker and Product indexes to evaluate weathering condition of Yellow Brown Forest soils in Japan: Geoderma, 130, 346-355.

United States Department of Agriculture (USDA), 1984, Procedures for collecting soil samples and methods of analysis for soil survey: Washington, D.C., United States Department of Agriculture, Soil Conservation Service, Soil Survey Investigation Report 1, 68 p.

United States Department of Agriculture (USDA), 2006, Keys to Soil Taxonomy: Washington, D.C., United States Department of Agriculture, Natural Resources Conservation Service, $332 \mathrm{p}$.

Waltman, S.W., Ciolkosz, E.J., 1995, Prairie soil development in Northwestern Pennsylvania: Soil Science, 160, 199-208.

World Reference Base for Soil Resources (WRB), 2006, World Reference Base for Soil Resources 2006 (World Soil Resources Reports No. 103): Roma, Food and Agriculture Organization of the United Nations, $132 \mathrm{p}$.

Yakimenko O.S., Sedov S.N., Solleiro-Rebolledo, E. 2007, The humus status of modern and buried volcanic soils in Mexico and its role in the paleogeographic interpretation of tephra-paleosol sequences: Eurasian Soil Science, 40, 274-280.

Manuscrito recibido: Diciembre 10, 2010

Manuscrito corregido recibido: Noviembre 15, 2011.

Manuscrito aceptado: Noviembre 15, 2011. 